\title{
Four-dimensional live imaging of hemodynamics in mammalian embryonic heart with Doppler optical coherence tomography
}

\author{
Shang Wang ${ }^{1}$, David S. Lakomy ${ }^{1}$, Monica D. Garcia ${ }^{1}$, Andrew L. Lopez III ${ }^{1}$, Kirill V. \\ Larin $^{1,2,3}$, and Irina V. Larina ${ }^{1, *}$ \\ ${ }^{1}$ Department of Molecular Physiology and Biophysics, Baylor College of Medicine, One Baylor \\ Plaza, Houston, TX 77030, U.S \\ 2Department of Biomedical Engineering, University of Houston, 3605 Cullen Blvd., 77204, \\ Houston, TX 77204, U.S \\ ${ }^{3}$ Interdisciplinary Laboratory of Biophotonics, Tomsk State University, Tomsk 634050, Russia
}

\section{Abstract}

Hemodynamic analysis of the mouse embryonic heart is essential for understanding the functional aspects of early cardiogenesis and advancing the research in congenital heart defects. However, high-resolution imaging of cardiac hemodynamics in mammalian models remains challenging, primarily due to the dynamic nature and deep location of the embryonic heart. Here we report four-dimensional micro-scale imaging of blood flow in the early mouse embryonic heart, enabling time-resolved measurement and analysis of flow velocity throughout the heart tube. Our method uses Doppler optical coherence tomography in live mouse embryo culture, and employs a postprocessing synchronization approach to reconstruct three-dimensional data over time at a $100 \mathrm{~Hz}$ volume rate. Experiments were performed on live mouse embryos at embryonic day 9.0. Our results show blood flow dynamics inside the beating heart, with the capability for quantitative flow velocity assessment in the primitive atrium, atrioventricular and bulboventricular regions, and bulbus cordis. Combined cardiodynamic and hemodynamic analysis indicates this functional imaging method can be utilized to further investigate the mechanical relationship between blood flow dynamics and cardiac wall movement, bringing new possibilities to study biomechanics in early mammalian cardiogenesis.

Four-dimensional live hemodynamic imaging of the mouse embryonic heart at embryonic day 9.0 using Doppler optical coherence tomography, showing directional blood flows in the sinus venosus, primitive atrium, atrioventricular region and vitelline vein.

\section{Graphical Abstract}

Congenital heart disease is the most common congenital disorder, affecting $1 \%$ newborns in the U.S. Visualization and measurement of hemodynamics in early developing mammalian heart can provide great insights for improved understanding of normal cardiogenesis and congenital cardiac

*Corresponding author: larina@bcm.edu, Phone: +01 7137983974.

Author biographies

Please see Supporting Information online. 
defects. In this study, Doppler optical coherence tomography is performed in live mouse embryo culture to obtain the first four-dimensional high-resolution imaging and measurement of hemodynamic features in the mammalian embryonic heart.

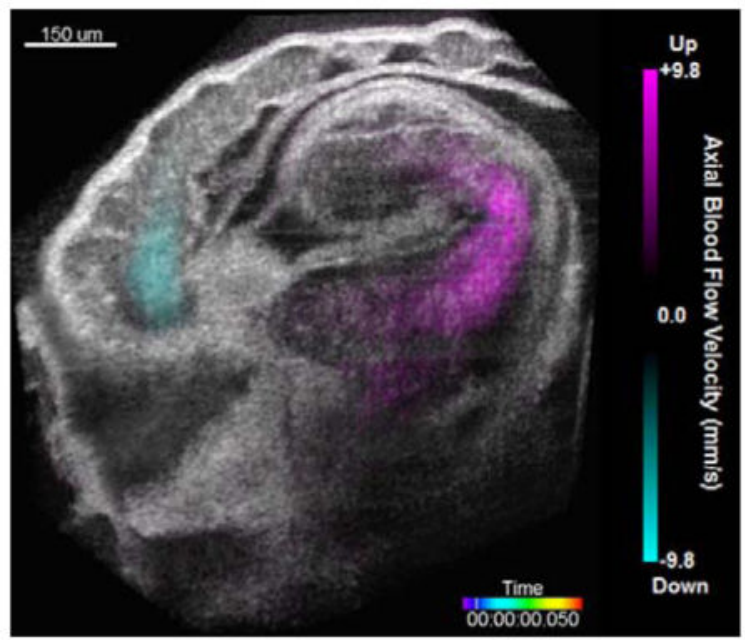

\section{Keywords}

Doppler optical coherence tomography; cardiac hemodynamics; live imaging; mouse embryo; retrograde blood flow

\section{Introduction}

Hemodynamics is a key biomechanical factor and plays a pivotal role in early cardiac morphogenesis [1,2] and cardiac conduction system development [3, 4]. Abnormal hemodynamics can lead to congenital heart disease [5, 6], which is the most common congenital disorder affecting about 40,000 newborns per year in the United States [7]. In spite of our understanding of the significant role of hemodynamics during cardiovascular development, the assessment of hemodynamics in the mammalian embryonic heart is very limited $[8,9]$, largely owing to the lack of proper functional imaging approaches with sufficient field of view and spatiotemporal resolution. The ability to visualize and measure blood flow in the embryonic heart at high resolution in a mammalian model remains a highly desired tool needed to advance the research and understanding of human congenital heart defects.

Acoustic imaging techniques, e.g. Doppler ultrasonic imaging, are widely used in the clinical environment to examine fetal cardiovascular health [10, 11]. However, in preclinical research using mouse models, the relatively poor spatial resolution (tens to hundreds of microns) of these techniques cannot provide sufficient resolving capability to obtain detailed hemodynamic information from inside the developing heart [12]. To achieve higher spatial resolution, confocal microscopy with vital fluorescent reporters is a powerful approach for imaging blood flow dynamics during the development of mouse embryonic vasculature [13, 14]. However, experiments are mainly limited to blood vessels from the extraembryonic yolk 
sac due to the lack of depthwise field of view [15, 16]. Nonlinear florescence microscopy, such as two-photon and multi-photon microscopy, which has a higher imaging depth than confocal microscopy, is becoming a popular tool in developmental biology [17, 18].

Although it was successfully applied for three-dimensional (3D) imaging of live mammalian embryos [19,20], applications were mainly limited to pre-implantation embryos, and no data from the embryonic heart has been reported. Thus, to meet the demand of probing detailed hemodynamic features in the mouse embryonic heart, an imaging approach with the spatial scale that fills the gap between traditional modalities is required.

Optical coherence tomography (OCT) is an emerging 3D embryonic imaging method with micro-scale spatial resolution and several millimetres penetration depth [21-23]. Doppler OCT, based on the optical phase from OCT complex signals, provides the capability of quantitatively mapping the velocities of moving scatters (such as blood cells) inside tissue [24-26]. Using Doppler OCT for live blood flow imaging has been established in various animal models, including Xenopus laevis [27], Drosophila [28], chick [29, 30], and mouse $[31,32]$. Since the embryonic heart is a highly dynamic organ, direct time-resolved highresolution imaging of hemodynamics inside the heart in $3 \mathrm{D}$ is challenging due to the limited volumetric imaging speed of OCT. To overcome this limitation, gated data acquisition and post-processing approaches have been proposed. Mariampillai et al utilized a second OCT system to probe cardiac motion as the gating signal for Doppler OCT imaging and obtained four-dimensional (4D) blood flow dynamics in the Xenopus laevis heart at a $45 \mathrm{~Hz}$ volume rate [33]. Jenkins et al applied an image-based retrospective gating algorithm [34] to measure 4D hemodynamics in the quail embryonic heart [35]. Additionally, with a similar post-processing approach [36], Liu et al reconstructed 4D blood flow dynamics in the chick embryonic outflow tract and characterized the biomechanics of the heart wall [37]. Despite these efforts, high-resolution imaging of hemodynamics in the mammalian embryonic heart has yet to be reported. Mice are the only mammalian organism that has a variety of genetic manipulation tools available for engineering mutants modelling human congenital heart defects [38]. Imaging 4D cardiac hemodynamics in the live mouse embryo can bring the knowledge that is translational and that will directly contribute to the understanding and the clinical care of congenital cardiac diseases [39], which is a major advantage over the avian and amphibian models.

In this paper, we report the first 4D live imaging and hemodynamic analysis in the mouse embryonic heart at a volume rate of $100 \mathrm{~Hz}$ and a spatial resolution of $\sim 5 \mu \mathrm{m}$. With Doppler OCT and post-processing synchronization, this method can provide detailed structural and functional information of early developing mammalian hearts and can indicate time-resolved blood flow patterns throughout the heart tube. In addition, combined with cardiodynamic analysis, our approach can be employed to assess the mechanical relationship between blood flow dynamics and cardiac wall movement. This work opens great opportunities to study the biomechanical aspects of the mouse embryonic heart during early development, which will advance our knowledge of the biomechanics in mammalian cardiogenesis. 


\section{Materials and Methods}

\subsection{OCT system}

We employed a home-built spectral-domain OCT system $[40,41]$ with a central wavelength of $808 \mathrm{~nm}$ and a bandwidth of $\sim 110 \mathrm{~nm}$. A fiber-based Michelson interferometer was utilized for the interference of light from reference and sample arms. The interference fringes were spatially resolved using a home-built spectrometer containing a diffraction grating, a focusing lens system and a line-field CMOS camera (spL4096-140km, Basler). The two-dimensional (2D) scanning of the imaging beam was obtained through two galvanometer mirrors. The system had an A-line rate of $\sim 68 \mathrm{kHz}$, and provided the spatial resolutions of $\sim 5 \mu \mathrm{m}$ (in tissue) in the axial direction and $\sim 4 \mu \mathrm{m}$ in the transverse direction. The system sensitivity was measured as $\sim 97 \mathrm{~dB}$ with a sensitivity drop of $\sim 4 \mathrm{~dB}$ over $\sim 1 \mathrm{~mm}$ in depth. The OCT sample arm was placed in an incubator during the entire imaging process. To generate high-resolution 4D images, data acquisition was conducted to have Bscans over time (for at least two heartbeat cycles) in a stepped parallel slice geometry at different spatial locations spanning the whole mouse embryonic heart [41].

\subsection{Mouse embryos}

Mouse embryos at embryonic day 9.0 (E9.0) with 12-16 somites were utilized in this study. At this developmental stage, looping of the heart initiates, forming four major parts: the sinus venosus, primitive atrium, primitive ventricle, and bulbus cordis. Mice were mated overnight, and females were monitored for a vaginal plug in the morning. The details of mouse embryo preparation can be found in our previous work [42]. Briefly, under $37^{\circ} \mathrm{C}$, embryos were dissected with the yolk sac intact in culture medium containing 89\% DMEM/ F12, 1\% penicillin-streptomycin and $10 \%$ fetal bovine serum. The embryos were then transferred to a humidified incubator (VWR Symphony) at $37^{\circ} \mathrm{C}$ with $5 \% \mathrm{CO}_{2}$ to recover for 1.5 hours. The OCT imaging of the mouse embryos was conducted inside a humidified incubator $\left(37^{\circ} \mathrm{C}, 5 \% \mathrm{CO}_{2}\right)$ where the sample arm of the OCT system is located. All animal manipulation procedures have been approved by the Institutional Animal Care and Use Committee of Baylor College of Medicine, and our experiments followed the approved guidelines and regulations.

\subsection{Doppler imaging and 4D reconstruction}

The data processing for 4D Doppler OCT hemodynamic imaging is illustrated in Figure 1. From the interpolated equal $k$-space interference fringes, fast Fourier transform was performed to obtain complex OCT signals $I$, where $I=A+i B$. The intensity of each pixel was calculated as

$$
|I|=\sqrt{A^{2}+B^{2}}
$$

which was mapped as the structural image with a logarithm scale. For Doppler processing, we applied the windowed Kasai autocorrelation function to estimate the blood flow velocity 
[43, 44]. The detailed derivation can be found in previous work on Doppler ultrasound [45]. The Doppler frequency shift $f$ from the spatial position $(m, n)$ was calculated as

$$
f=\frac{f_{a}}{2 \pi} \Delta \varphi=\frac{f_{a}}{2 \pi} \arctan \frac{\sum_{m=1}^{M} \sum_{n=1}^{N-1}\left(A_{m, n+1} B_{m, n}-B_{m, n+1} A_{m, n}\right)}{\sum_{m=1}^{M} \sum_{n=1}^{N-1}\left(A_{m, n+1} A_{m, n}+B_{m, n+1} B_{m, n}\right)}
$$

where $f_{a}$ is the A-line rate of the OCT system, $\Delta \varphi$ is the averaged phase shift between Alines, $M \times N$ is the size of the window with $M$ and $N$ representing the number of pixels in the axial and transverse directions, respectively, and $m$ and $n$ are the respective axial and transverse pixel positions. With the Doppler frequency $f$ available, the absolute flow velocity $v$ and the axial flow velocity $v_{a}$ can be quantified as

$$
v=\frac{v_{a}}{\cos \theta}=\frac{\lambda f}{2 n \cos \theta}
$$

where $\lambda$ is the central wavelength of the OCT system, $n$ is the refractive index of blood (assumed as 1.4), and $\theta$ is the angle between the blood flow direction and the OCT imaging beam, which was measured based on the 3D OCT structural image. To determine the blood flow direction, a vertical 2D cross-sectional view through the structural OCT volume along the heart tube was created at each measurement point. A line through the centre of the heart tube in the 2D image was used to represent the blood flow direction, and the angle between this line and the vertical direction was manually measured as $\theta$. The mapping of the axial flow velocity to the spatial position produced a 2D Doppler image.

The post-processing synchronization for the B-scan time lapse to have the same phase of heartbeat cycle was conducted based on the structural images, as previously reported [41, $46,47]$. The 4D structural synchronization provides the information of the temporal and spatial locations to rearrange the corresponding 2D Doppler images, enabling 4D reconstruction of the cardiac blood flow. The registration of the dynamic structural images and blood flow images was used as the 4D hemodynamic imaging result for the mouse embryonic hearts.

\section{Results}

Four-dimensional cardiac hemodynamic imaging in an E9.0 mouse embryo is shown in Figure 2, Media 1 and Media 2, with two cross-sectional views covering the sinus venosus, primitive atrium, primitive ventricle and bulbus cordis of the heart. With 4D visualization, the features of the blood flow dynamics are clearly distinguishable. As shown in Figure 2(A), as the atrium fully opens, a strong flow forms and feeds into the ventricle through the sinus venosus and the atrium. Since blood flows into the sinus venosus from two conjoining veins, in the atrium two separate streams of blood flow can be visualized in Figure 2(B). 
When the primitive atrium contracts, retrograde flow forms inside the atrium, as indicated with the solid arrows in Figure 2(C) and Figure 2(D). Such backward flow is also present in the vitelline vein. Similar to the atrium, when the bulbus cordis is wide-open, strong blood output forms through the bulbus cordis, as shown in Figure 2(F). During the ventricle contraction, from Figure 2(G), it can be seen that blood flows from the ventricle to the bulbus cordis, with the Doppler signals present in the bulboventricular region. Concurrently, retrograde flow is forming in the atrioventricular region (pointed with a dashed arrow), which indicates the presence of a simultaneous bi-directional flow inside the primitive ventricle at this time point. As the bulbus cordis contracts, retrograde flow appears in the bulbus cordis and bulboventricular region, as indicated with the dotted arrows in Figure $2(\mathrm{H})$, where retrograde flow in the atrioventricular region is still present (pointed with a dashed arrow). During further contraction of the bulbus cordis, retrograde flow remains in the bulboventricular region (indicated with a dotted arrow), shown in Figure 2(E). At the same time, the ventricle starts to open, and strong blood flow entering the ventricle is visualized, thus creating another time point when simultaneous bi-directional flow is present in the primitive ventricle. To the best of our knowledge, these results are the first highresolution 4D visualizations of hemodynamic flow patterns inside the mouse embryonic heart tube, and can be of great value for the further studies of biomechanics during early mammalian heart development.

A cross-section in the atrioventricular region of the embryonic heart was taken, and the absolute blood flow velocity was mapped over the spatial region within the heart tube, as shown in Figure 3. With the 2D smoothed surface in Figure 3(A), it can be seen that the blood forms a laminar flow over the spatial region, which is also indicated by the parabolic fit of the one-dimensional velocity profile in Figure 3(B). The results of peak blood flow velocity in the embryonic heart are comparable with the range of hemodynamic measurements in the mouse embryos from other developmental stages measured in utero using Doppler ultrasonic imaging [48-50]. Also, our measured velocity values are comparable to those from the chick embryonic heart obtained with OCT [30, 51]. These data suggest the feasibility of our approach to perform quantitative 2D spatially-resolved measurements of the absolute blood flow velocity inside the mouse embryonic heart.

Time-resolved analysis of the peak flow velocity at four selected locations throughout the heart tube of an E9.0 mouse embryo is shown in Figure 4. The positions where the velocity measurements were taken are labelled in 3D with yellow plates, shown in Figure 4(A-D). These data clearly show that retrograde flows are present at all four positions, including the primitive atrium, atrioventricular and bulboventricular regions, and bulbus cordis. From Figure 4(E), in the primitive atrium, the inflow, central and outflow regions have blood flow turning from forward to retrograde flow at close but different time points, indicated with three short green arrows. For retrograde flows, the outflow region has the maximum velocity later than the inflow region, which is labelled with the solid and the dashed orange arrows. Due to the closure of the atrium central region, the retrograde flow at this location undergoes a decrease in velocity, shown with a dotted orange arrow. The velocity values that are still present at this time are from the Doppler (phase) signal noise. Together with Figure 4(F), it can be seen that the time points at which the flow direction switches show delays from the atrioventricular region as compared to the central region of the primitive atrium. The delays 
are measured as $\sim 40 \mathrm{~ms}$ and $\sim 10 \mathrm{~ms}$ for the forward to retrograde switch and the retrograde to forward switch, respectively. From Figure 4(F) and Figure 4(G), the previously visualized simultaneous bi-directional blood flows in the primitive ventricle are quantitatively shown with the velocity profiles in the atrioventricular and bulboventricular regions, which are labelled with yellow blocks. In Figure 4(G) and Figure 4(H), the bulbus cordis shows no time delay from the bulboventricular region for the switch of flow direction, while the appearing of forward flow is ahead of $\sim 30 \mathrm{~ms}$ in the bulbus cordis as compared to the bulboventricular region. These time-resolved analyses confirm the 4D observations (Figure 2, Media 1 and Media 2) and demonstrate the capability of our imaging approach to provide quantitative assessment of specific cardiac blood flow dynamics in mouse embryos with a high temporal resolution.

During mammalian heart development, dramatic morphological changes occur in the bulboventricular region, e.g. the formation of the right ventricle and the interventricular septum $[52,53]$. To demonstrate the feasibility of the described method for studying the biomechanical relationship between blood flow and heart wall movement, we analysed the hemodynamic features in the bulboventricular region in relation to the wall movements from the central parts of the ventricle and the bulbus cordis, shown in Figure 5. Cardiac wall dynamics were measured with diameter changes of the heart tube at specific locations based on 4D structural OCT imaging. In Figure 5(A), the primitive ventricle and the bulbus cordis show similar contraction and relaxation profiles over time with a small temporal difference of about 10-20 ms. Also, strong forward bulboventricular blood flow occurs during the contraction of both the ventricle and the bulbus cordis. Retrograde flow forms near the end of the contraction. Specifically, retrograde flow starts when the ventricle is at its most contracted state while the bulbus cordis is still in the process of contraction (close to the end of the cycle). After this, retrograde flow lasts for a short period during the initial relaxation of both the bulbus cordis and the ventricle. These observations can be seen in the 4D visualization (Figure 2 and Media 2). However, such time-resolved quantitative plots provide greater details, helping to identify a clearer temporal relationship.

As a demonstration of further analysis that can be performed based on this functional imaging approach, we analysed the heart wall velocity as a function of time, which is shown in Figure 5(B). The heart wall velocity was estimated as a half of the derivative of the smoothed diameter over time. These plots show that the start of bulboventricular retrograde flow occurs at a time close to the maximum contraction velocity of the bulbus cordis wall (pointed by a solid arrow). During this retrograde flow, the relaxation velocity of the ventricle wall reaches its highest value (pointed with a dotted arrow). These suggest that the bulbus cordis contraction velocity and the ventricle relaxation velocity are possibly two of the contributing factors for the formation and maintenance of retrograde flow in the bulboventricular region. Also, the strong forward blood flow is coincident with the maximum wall contraction velocity of the primitive ventricle (pointed with a dashed arrow), implying a possible correlation between blood flow dynamics and the velocity of the heart wall movement. These results indicate that the described 4D imaging method can provide combined cardiodynamic and hemodynamic analysis over time, which will help to achieve improved understanding of the biomechanics in early mammalian embryonic heart. 
With a total of six mouse embryos at E9.0 (12-16 somites), Figure 6 shows the maximum of peak flow velocity over time from the bulboventricular region for each embryo. The heartbeat cycle of these embryos is $0.49 \pm 0.04 \mathrm{~s}$. For the forward and the backward flows, the maximum velocities are measured as $7.7 \pm 2.8 \mathrm{~mm} / \mathrm{s}$ and $9.5 \pm 3.1 \mathrm{~mm} / \mathrm{s}$, respectively. The variation in velocity between embryos might due to individual developmental differences.

\section{Discussions and Conclusions}

Our results demonstrate that this Doppler-OCT-based 4D hemodynamic imaging approach is able to provide high-resolution visualization and measurement of blood flow dynamics throughout the early developing heart tube in mouse embryos, and can be utilized to perform time-resolved analysis of the mechanical relationship between blood flow dynamics and heart wall movement. This study opens the door for a variety of experiments to investigate biomechanical factors in early mammalian cardiogenesis, which can lead to advances in research on congenital heart disease.

Our Doppler OCT imaging was based on a windowed Kasai autocorrelation function. It was demonstrated that the velocity estimation with the Kasai autocorrelation function provides greater numerical efficiency compared with directly computing the phase difference between A-lines [43]. In our computation, we applied a window size of $10 \times 10$ pixels. Applying larger window size was found to have improved accuracy of velocity assessment [43], however, at the expense of increased processing time and blurring of final images. The output of phase calculation in Equation (2) has the range from $-\pi$ to $+\pi$, thus limiting the highest measurable axial flow velocity as $\sim 9.8 \mathrm{~mm} / \mathrm{s}$. With an angle $\theta$ existing between the directions of blood flow and OCT imaging beam, the measurable range of absolute blood flow velocity is proportionally extended by a factor of $1 / \cos \theta$. Also, with a higher A-line rate, higher velocity could be measured without aliasing of optical phase signals. The sensitivity of the axial velocity measurement is mainly determined by the stability of phase from the OCT system during embryonic imaging. In our case, the lowest axial velocity that can be resolved is $\sim 0.5 \mathrm{~mm} / \mathrm{s}$. Better phase stability could be achieved by implementing a common-path configuration of the low coherence interferometer [54, 55], which will lead to an improved sensitivity of velocity.

The manual measurement of the angle between laser beam and blood flow direction in this work can be time consuming for a large amount of data analysis and might have influence on the quantification of flow velocity. A very recent study on Doppler OCT proposed an approach for automatic segmentation and skeletonization of blood vessels [56], which potentially can be integrated with our 4D hemodynamic imaging approach and employed for automatic extraction of the flow direction in the mouse embryonic heart tube. This would provide a more robust and efficient way for quantitative analysis of the cardiac blood flow in the early mammalian hearts.

Data acquisition for the presented 4D OCT imaging approach takes 5 minutes to cover the whole embryonic heart with a micro-scale spatial sampling interval, and data synchronization is required through post-processing for $4 \mathrm{D}$ visualization. Direct volumetric imaging of a beating embryonic heart can significantly improve the efficiency of the mouse 
phenotyping process [57-59]. However, with the current speed of a state-of-the-art ultrafast swept laser source, the spatial sampling resolution of direct 4D imaging cannot be comparable to the results presented in this paper, causing lower quality of visualization, measurement and analysis. With further development in the fields of lasers and electronics, high-resolution real-time structural and hemodynamic imaging of live mouse embryonic heart could be possible.

The mouse embryonic heart starts to beat and incorporate blood into circulation at early E8.5. Since the OCT imaging depth is limited to several millimetres, and is associated with a sensitivity drop over depth, the developmental period of embryo that could be covered by the presented imaging procedure is from early E8.5 to late E9.5 with the yolk sac intact. A majority of cardiac morphological and hemodynamic changes occur during this period, offering great opportunities to study early cardiogenesis. For later stages, cardiac OCT imaging can be achieved with the yolk sac partially removed [60], and the embryos can be kept alive with roller culture techniques [61], extending the potential stages for the investigation of mammalian heart development. Applying a longer wavelength of laser (e.g. $1300 \mathrm{~nm}$ ) in the OCT system could provide an improved depth of view to probe deeper inside the embryo [62].

The experiments reported here were performed in static mouse embryo culture. While in this approach the embryos are removed from the uterus and are not in a perfectly natural environment, there are currently no methods to visualize the beating mouse embryonic heart in utero at this spatial and temporal resolution. Based on the previous studies, the early-stage (E7.5-E10.5) mouse embryos cultured on the imaging stage for 24 hours exhibit similar developmental changes as those in normal pregnancy [63-65]. Thus, static culture enables otherwise inaccessible information of the live mouse embryo. The protocols of the static mouse embryo culture are well established [66-68] and are applied in combination with confocal microscopy by other groups [14, 69] as well as with OCT by our group [41, 59]. Although this culture method has been extensively utilized for cardiovascular analysis in mouse embryos, the measurement acquired through this approach should be taken cautiously since the embryos are not in their maternal environment. The static embryo culture is optimal for E7.5-E10.5 mouse developmental stages.

Retrograde flows in the early embryonic heart were previously reported in zebrafish and avian models [51, 70, 71]. With confocal imaging of zebrafish embryos, Vermot et al found that retrograde flow acted as an important biomechanical factor regulating the valve development in the early embryonic heart [72]. In our study, using Doppler OCT, we have presented the first 4D imaging and analysis of retrograde flows throughout the mouse embryonic heart. Based on the 4D structural and functional imaging results, it can be found that the retrograde flows have strong peak flow velocities that are comparable to the forward flows (Figure 4 and Figure 6), but occur at the time points when the diameter of heart tube is relatively small (Media 1, Media 2 and Figure 5). This suggests that the retrograde flows inside the embryonic heart could produce relatively higher shear stress on the cardiac wall, providing strong mechanical stimuli to the endocardium [72]. The quantitative results shown in this paper can serve as the basis for further investigation of blood flow dynamics during mammalian cardiac development. With high-resolution image-guided manipulation methods 
available for mouse embryos [73], this dynamic imaging approach can be implemented to study biomechanical and genetic regulations of the early mammalian heart development.

\section{Supplementary Material}

Refer to Web version on PubMed Central for supplementary material.

\section{Acknowledgments}

This work is supported by the National Institute of Health with grants R01HL120140, U54HG006348 and R25GM056929, and by the Optical Imaging and Vital Microscopy Core at Baylor College of Medicine.

\section{References}

1. Hove JR, Koster RW, Forouhar AS, Acevedo-Bolton G, Fraser SE, Gharib M. Nature. 2003; 421:172-177. [PubMed: 12520305]

2. Boselli F, Freund JB, Vermot J. Cellular and molecular life sciences : CMLS. 2015; 72:2545-2559. [PubMed: 25801176]

3. Reckova M, Rosengarten C, deAlmeida A, Stanley CP, Wessels A, Gourdie RG, Thompson RP, Sedmera D. Circulation research. 2003; 93:77-85. [PubMed: 12775585]

4. Hall CE, Hurtado R, Hewett KW, Shulimovich M, Poma CP, Reckova M, Justus C, Pennisi DJ, Tobita K, Sedmera D, Gourdie RG, Mikawa T. Development. 2004; 131:581-592. [PubMed: 14711873]

5. Peskin CS, Tu C. Computers in biology and medicine. 1986; 16:331-359. [PubMed: 3769465]

6. Taber LA. Prog Biophys Mol Biol. 1998; 69:237-255. [PubMed: 9785941]

7. Hoffman JI, Kaplan S. J Am Coll Cardiol. 2002; 39:1890-1900. [PubMed: 12084585]

8. Furst, B. The Heart and Circulation. Springer; London: 2014. Hemodynamics of the Early Embryo Circulation; p. 21-29.

9. Hove JR. Pediatr Res. 2006; 60:6-13. [PubMed: 16690955]

10. Chang C-H, Chang F-M, Yu C-H, Liang R-I, Ko H-C, Chen H-Y. Ultrasound in Medicine \& Biology. 2000; 26:777-785. [PubMed: 10942825]

11. Hecher K, Campbell S, Doyle P, Harrington K, Nicolaides K. Circulation. 1995; 91:129-138. [PubMed: 7805194]

12. Stypmann J. Echocardiography. 2007; 24:97-112. [PubMed: 17214632]

13. Larina IV, Shen W, Kelly OG, Hadjantonakis AK, Baron MH, Dickinson ME. Anatomical record (Hoboken, NJ : 2007). 2009; 292:333-341.

14. Lucitti JL, Jones EA, Huang C, Chen J, Fraser SE, Dickinson ME. Development. 2007; 134:33173326. [PubMed: 17720695]

15. Garcia MD, Larina I. Frontiers in Physiology. 2014; 5

16. Lopez, A., III; Garcia, M.; Dickinson, M.; Larina, I. Live Confocal Microscopy of the Developing Mouse Embryonic Yolk Sac Vasculature. In: Ribatti, D., editor. Vascular Morphogenesis. Springer; New York: 2015. p. 163-172.

17. Supatto W, Truong TV, Debarre D, Beaurepaire E. Current opinion in genetics \& development. 2011; 21:538-548. [PubMed: 21917444]

18. Truong TV, Supatto W, Koos DS, Choi JM, Fraser SE. Nat Meth. 2011; 8:757-760.

19. McDole K, Xiong Y, Iglesias PA, Zheng Y. Developmental Biology. 2011; 355:239-249. [PubMed: 21539832]

20. Squirrell JM, Wokosin DL, White JG, Bavister BD. Nature biotechnology. 1999; 17:763-767.

21. Karunamuni G, Gu S, Peterson LM, Ma P, Wang YT, Rollins AM, Jenkins MW, Watanabe M. Frontiers in Physiology. 2014; 5:351. [PubMed: 25309451]

22. Yelbuz TM, Choma MA, Thrane L, Kirby ML, Izatt JA. Circulation. 2002; 106:2771-2774. [PubMed: 12451001] 
23. Jenkins MW, Rothenberg F, Roy D, Nikolski VP, Hu Z, Watanabe M, Wilson DL, Efimov IR, Rollins AM. Optics Express. 2006; 14:736-748. [PubMed: 19503392]

24. Wang XJ, Milner TE, Nelson JS. Optics Letters. 1995; 20:1337-1339. [PubMed: 19859518]

25. Zhao Y, Chen Z, Saxer C, Xiang S, de Boer JF, Nelson JS. Optics Letters. 2000; 25:114-116. [PubMed: 18059800]

26. Leitgeb RA, Werkmeister RM, Blatter C, Schmetterer L. Progress in Retinal and Eye Research. 2014; 41:26-43. [PubMed: 24704352]

27. Yazdanfar S, Kulkarni M, Izatt J. Optics Express. 1997; 1:424-431. [PubMed: 19377566]

28. Zurauskas M, Bradu A, Ferguson DR, Hammer DX, Podoleanu A. Journal of Biophotonics. 2015:n/a-n/a.

29. Davis AM, Rothenberg FG, Shepherd N, Izatt JA. Journal of the Optical Society of America. A, Optics, image science, and vision. 2008; 25:3134-3143.

30. Davis A, Izatt J, Rothenberg F. The Anatomical Record: Advances in Integrative Anatomy and Evolutionary Biology. 2009; 292:311-319.

31. Larina IV, Sudheendran N, Ghosn M, Jiang J, Cable A, Larin KV, Dickinson ME. J Biomed Opt. 2008; 13:060506. [PubMed: 19123647]

32. Larina IV, Ivers S, Syed S, Dickinson ME, Larin KV. Opt Lett. 2009; 34:986-988. [PubMed: 19340193]

33. Mariampillai A, Standish BA, Munce NR, Randall C, Liu G, Jiang JY, Cable AE, Vitkin IA, Yang VXD. Optics Express. 2007; 15:1627-1638. [PubMed: 19532397]

34. Gargesha M, Jenkins MW, Wilson DL, Rollins AM. Opt Express. 2009; 17:10786-10799. [PubMed: 19550478]

35. Jenkins MW, Peterson L, Gu S, Gargesha M, Wilson DL, Watanabe M, Rollins AM. Journal of Biomedical Optics. 2010; 15:066022-066022-066024. [PubMed: 21198196]

36. Liu A, Wang R, Thornburg KL, Rugonyi S. J Biomed Opt. 2009; 14:044020. [PubMed: 19725731]

37. Liu A, Yin X, Shi L, Li P, Thornburg KL, Wang R, Rugonyi S. PLoS ONE. 2012; 7:e40869. [PubMed: 22844414]

38. Moon A. Current topics in developmental biology. 2008; 84:171-248. [PubMed: 19186245]

39. Yutzey KE, Robbins J. Circulation. 2007; 115:792-799. [PubMed: 17296868]

40. Wang S, Lopez AL, Morikawa Y, Tao G, Li J, Larina IV, Martin JF, Larin KV. Biomedical Optics Express. 2014; 5:1980-1992. [PubMed: 25071943]

41. Lopez AL III, Wang S, Larin KV, Overbeek PA, Larina. Journal of Biomedical Optics. 2015; 20:090501-090501. [PubMed: 26385422]

42. Garcia, MD.; Lopez, AL., III; Larin, KV.; Larina, IV., editors. Imaging of Cardiovascular Development in Mammalian Embryos Using Optical Coherence Tomography. Springer; 2014.

43. Yang V, Gordon M, Qi B, Pekar J, Lo S, Seng-Yue E, Mok A, Wilson B, Vitkin I. Optics Express. 2003; 11:794-809. [PubMed: 19461792]

44. Yang VXD, Gordon ML, Mok A, Zhao Y, Chen Z, Cobbold RSC, Wilson BC, Alex Vitkin I. Optics Communications. 2002; 208:209-214.

45. Jensen, JA. Estimation of Blood Velocities Using Ultrasound: A Signal Processing Approach. Cambridge University Press; 1996.

46. Liebling M, Forouhar AS, Gharib M, Fraser SE, Dickinson ME. J Biomed Opt. 2005; 10:054001. [PubMed: 16292961]

47. Larin KV, Larina IV, Liebling M, Dickinson ME. Journal of Innovative Optical Health Sciences. 2009; 02:253-259.

48. Aristizábal O, Christopher DA, Foster FS, Turnbull DH. Ultrasound in Medicine \& Biology. 1998; 24:1407-1417. [PubMed: 10385963]

49. Ji RP, Phoon CKL, Aristizábal O, McGrath KE, Palis J, Turnbull DH. Circulation research. 2003; 92:133-135. [PubMed: 12574139]

50. Phoon CK, Turnbull DH. Physiological genomics. 2003; 14:3-15. [PubMed: 12824473]

51. Midgett M, Chivukula VK, Dorn C, Wallace S, Rugonyi S. Journal of The Royal Society Interface. 2015; 12 
52. Matitiau A, Geva T, Colan SD, Sluysmans T, Parness IA, Spevak PJ, Van Der Velde M, Mayer JE, Sanders SP. Journal of the American College of Cardiology. 1992; 19:142-148. [PubMed: 1370303]

53. Anderson RH, Becker AE, Wilkinson JL, Gerlis LM. British heart journal. 1976; 38:558-572. [PubMed: 1275986]

54. Kennedy BF, Malheiro FG, Chin L, Sampson DD. Journal of Biomedical Optics. 2014; 19:076006-076006. [PubMed: 25003754]

55. Joo C, Akkin T, Cense B, Park BH, de Boer JF. Optics Letters. 2005; 30:2131-2133. [PubMed: 16127933]

56. Qi L, Zhu J, Hancock AM, Dai C, Zhang X, Frostig RD, Chen Z. Biomedical Optics Express. 2016; 7:601-615. [PubMed: 26977365]

57. Jenkins MW, Adler DC, Gargesha M, Huber R, Rothenberg F, Belding J, Watanabe M, Wilson DL, Fujimoto JG, Rollins AM. Optics Express. 2007; 15:6251-6267. [PubMed: 19546930]

58. Yelin R, Yelin D, Oh WY, Yun SH, Boudoux C, Vakoc BJ, Bouma BE, Tearney GJ. J Biomed Opt. 2007; 12:064021. [PubMed: 18163837]

59. Wang S, Singh M, Lopez AL, Wu C, Raghunathan R, Schill A, Li J, Larin KV, Larina IV. Opt Lett. 2015; 40:4791-4794. [PubMed: 26469621]

60. Luo W, Marks DL, Ralston TS, Boppart SA. J Biomed Opt. 2006; 11:021014. [PubMed: 16674189]

61. Martin, P.; Cockroft, D. Culture of Postimplantation Mouse Embryos. In: Sharpe, P.; Mason, I., editors. Molecular Embryology. Humana Press; 2009. p. 7-22.

62. Ishida S, Nishizawa N. Biomedical Optics Express. 2012; 3:282-294. [PubMed: 22312581]

63. Jones EAV, Crotty D, Kulesa PM, Waters CW, Baron MH, Fraser SE, Dickinson ME. genesis. 2002; 34:228-235. [PubMed: 12434332]

64. Jones EAV, Baron MH, Fraser SE, Dickinson ME. American Journal of Physiology - Heart and Circulatory Physiology. 2004; 287:H1561-H1569. [PubMed: 15155254]

65. Jones, EAV.; Baron, MH.; Fraser, SE.; Dickinson, ME. Dynamic In Vivo Imaging of Mammalian Hematovascular Development Using Whole Embryo Culture. In: Baron, MH., editor. Developmental Hematopoiesis: Methods and Protocols. Humana Press; Totowa, NJ: 2005. p. 381-393.

66. Garcia MD, Udan RS, Hadjantonakis A-K, Dickinson ME. Cold Spring Harbor Protocols. 2011; 2011 pdb.prot5594.

67. Larina IV, Garcia MD, Vadakkan TJ, Larin KV, Dickinson ME. Cold Spring Harb Protoc. 2012; 2012:1035-1043. [PubMed: 23028074]

68. Garcia MD, Udan RS, Hadjantonakis AK, Dickinson ME. Cold Spring Harbor Protocols. 2011; 2011 pdb.prot5595.

69. Nowotschin S, Ferrer-Vaquer A, Hadjantonakis AK. Methods in enzymology. 2010; 476:351-377. [PubMed: 20691876]

70. Liebling M, Forouhar AS, Wolleschensky R, Zimmermann B, Ankerhold R, Fraser SE, Gharib M, Dickinson ME. Developmental Dynamics. 2006; 235:2940-2948. [PubMed: 16921497]

71. Freund JB, Goetz JG, Hill KL, Vermot J. Development. 2012; 139:1229-1245. [PubMed: 22395739]

72. Vermot J, Forouhar AS, Liebling M, Wu D, Plummer D, Gharib M, Fraser SE. PLoS Biol. 2009; 7:e1000246. [PubMed: 19924233]

73. Syed SH, Coughlin AJ, Garcia MD, Wang S, West JL, Larin KV, Larina IV. Journal of Biomedical Optics. 2015; 20:051020-051020. [PubMed: 25581495] 

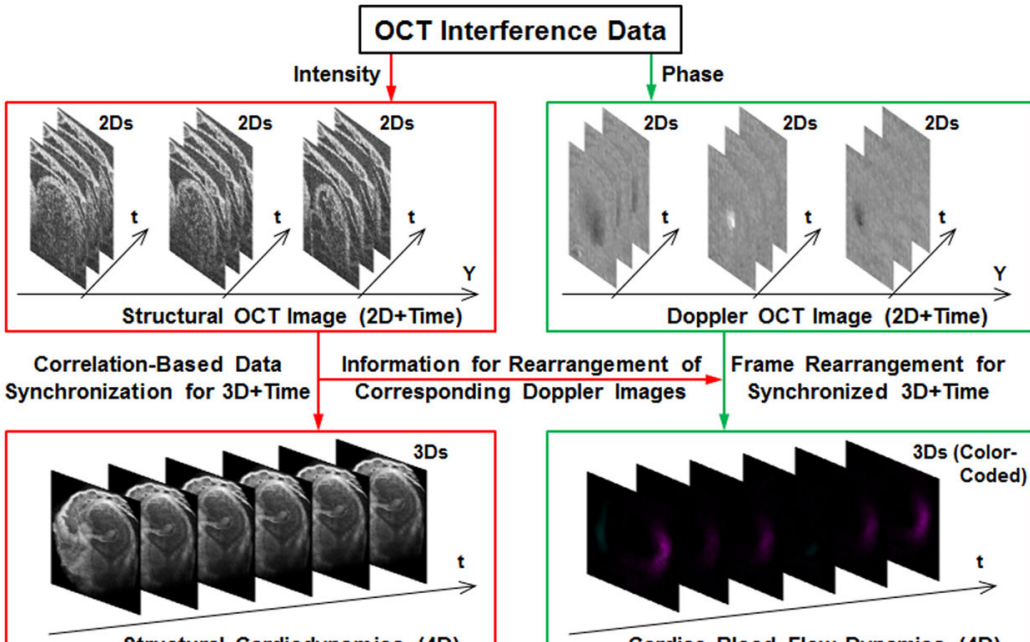

Doppler OCT Image (2D+Time)

earrangement of Frame Rearrangement for Synchronized 3D+Time Registration of 4D Structural and Blood Flow
Imaging of Early Mouse Embryonic Heart
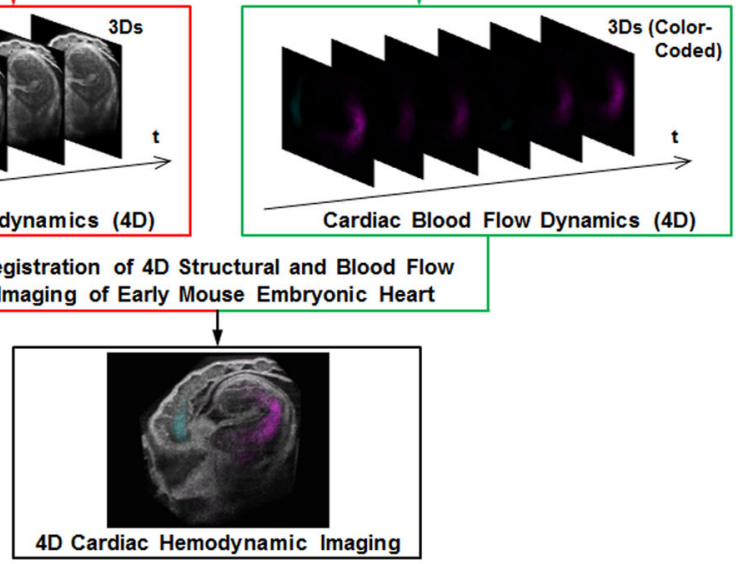

Figure 1.

Illustration of image processing for reconstruction of 4D hemodynamics and cardiodynamics from early-stage mouse embryonic heart. 


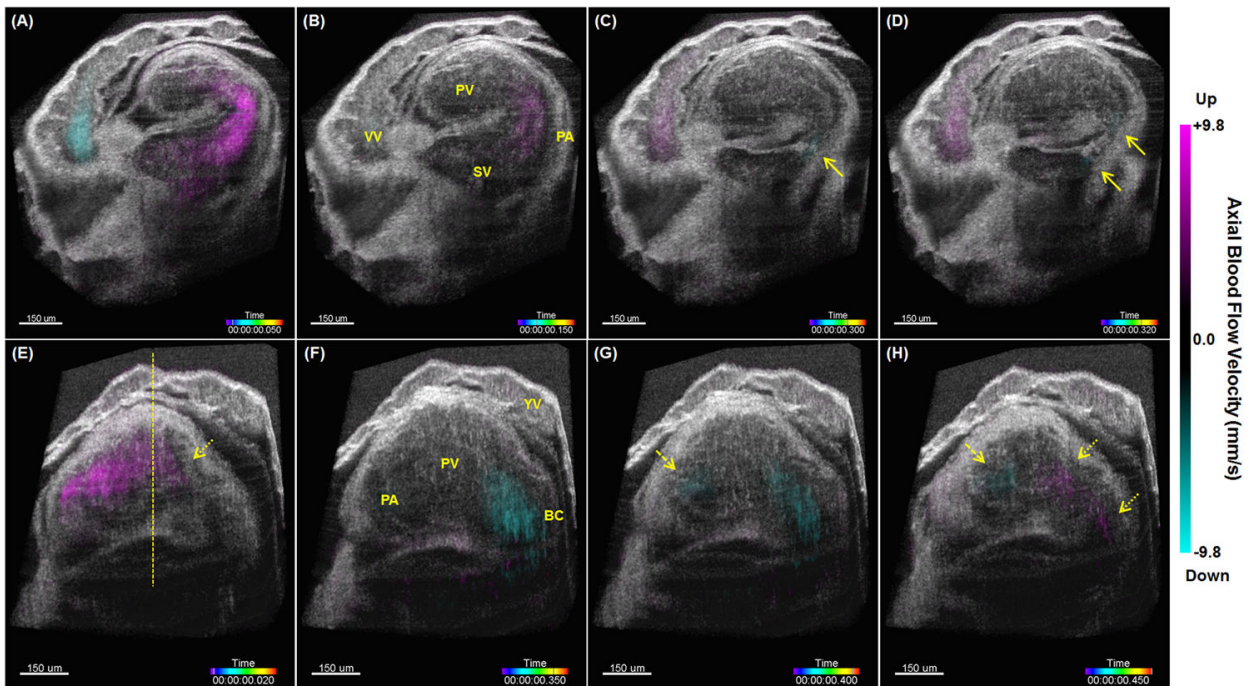

Figure 2.

Snapshots from 4D hemodynamic imaging of an E9.0 mouse embryonic heart, showing features of blood flow dynamics (Media 1 and Media 2). (A-D) are cross-sectional views that focus on the sinus venosus, primitive atrium and vitelline vein at different time points of cardiac cycle. $(\mathrm{E}-\mathrm{H})$ are cross-sectional views that focus on the primitive ventricle and bulbus cordis at different time points of cardiac cycle. Solid arrows point at retrograde flows in the primitive atrium. Dashed arrows point at retrograde flows in the atrioventricular region. Dotted arrows point at retrograde flows in the bulbus cordis and the bulboventricular region. The dashed line in (E) shows the location that has the highest axial position. YV: yolk sac vessel; VV: vitelline vein; SV: sinus venosus; PA: primitive atrium; PV: primitive ventricle; $\mathrm{BC}$ : bulbus cordis. 
(A)

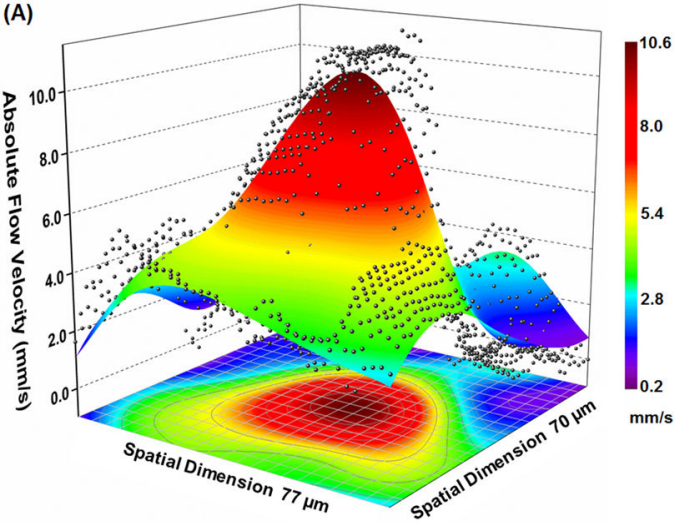

(B)

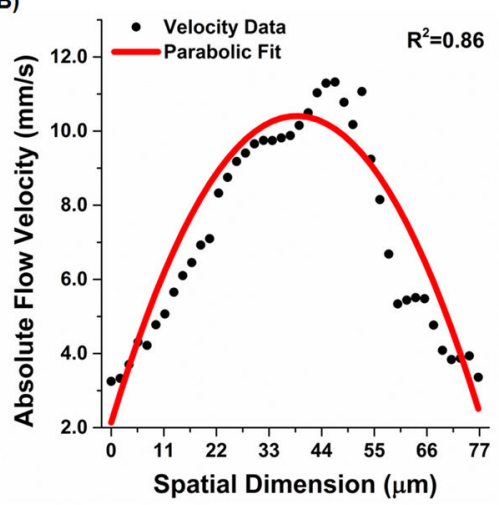

Figure 3.

Spatial profile of absolute blood flow velocity from the atrioventricular region of an E9.0 mouse embryonic heart. (A) 2D scatter plot of the spatial velocity distribution with a smoothed surface. (B) Parabolic fit of a one-dimensional spatial velocity profile. The spatial location where the velocity measurement is taken is labelled with a yellow plate in Figure 4(B). 

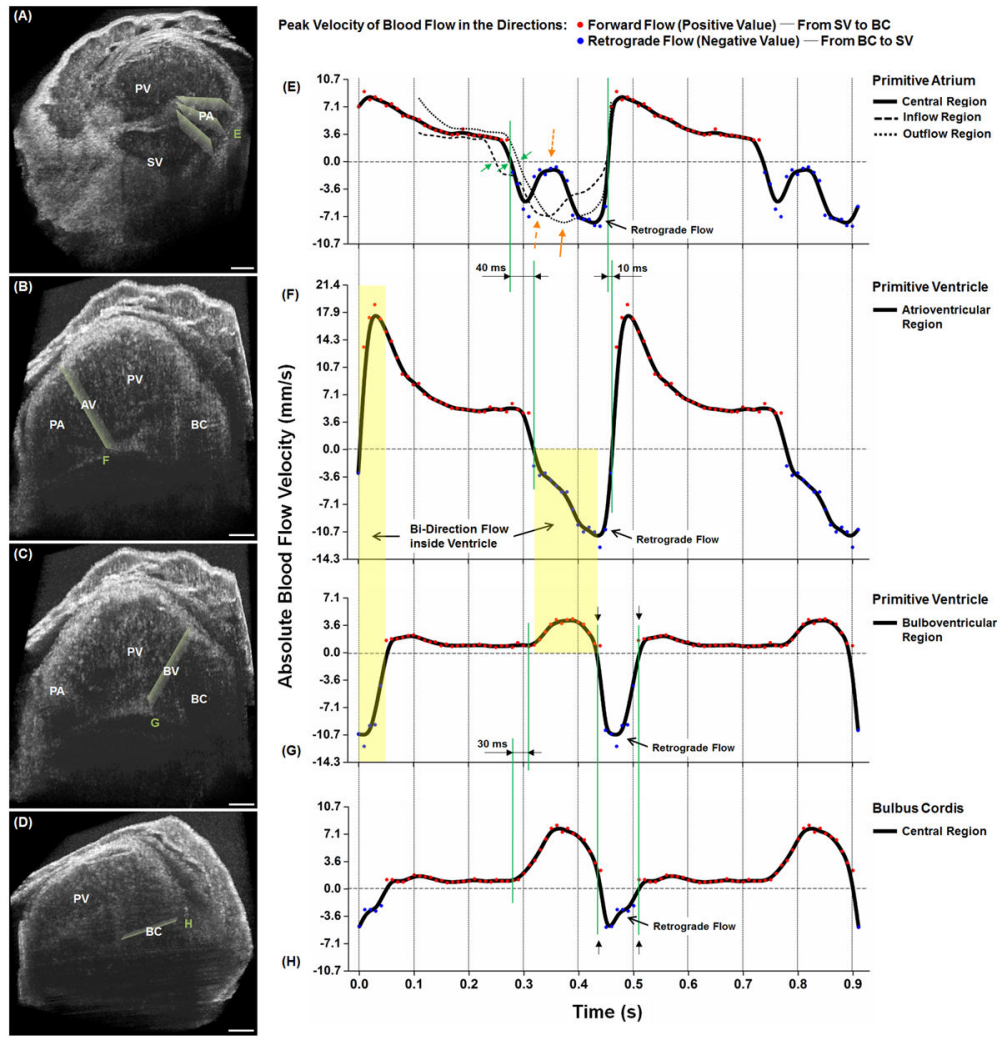

Figure 4.

Time-resolved analysis of the peak blood flow velocity throughout an E9.0 mouse embryonic heart tube indicates hemodynamic features of early mammalian heart. 3D OCT structural images with cross-sectional plates indicate the velocity measurement locations in the (A) primitive atrium, (B) atrioventricular region, (C) bulboventricular region and (D) bulbus cordis. The plate in (B) also indicates the location for the measurement of spatial flow velocity distribution in Figure 3. Scale bars correspond to $100 \mu \mathrm{m}$. Peak velocity profiles over time at the cardiac positions of the (E) primitive atrium, $(\mathrm{F})$ atrioventricular region, $(\mathrm{G})$ bulboventricular region, and $(\mathrm{H})$ bulbus cordis. SV: sinus venosus; PA: primitive atrium; PV: primitive ventricle; $\mathrm{BC}$ : bulbus cordis; $\mathrm{AV}$ : atrioventricular; $\mathrm{BV}$ :

bulboventricular. The second heartbeat cycle shown in $(\mathrm{E}-\mathrm{H})$ is duplication of the first one for better visualization. The green arrows in (E) point at the time points when the blood flow switches from forward to retrograde flow at three locations of the primitive atrium. The dashed and solid orange arrows point at the peak of retrograde flow at the inflow and outflow region of the atrium. The dotted orange arrow points at the decrease of the retrograde flow velocity at the atrium central region due to the closure of heart tube at this position. 

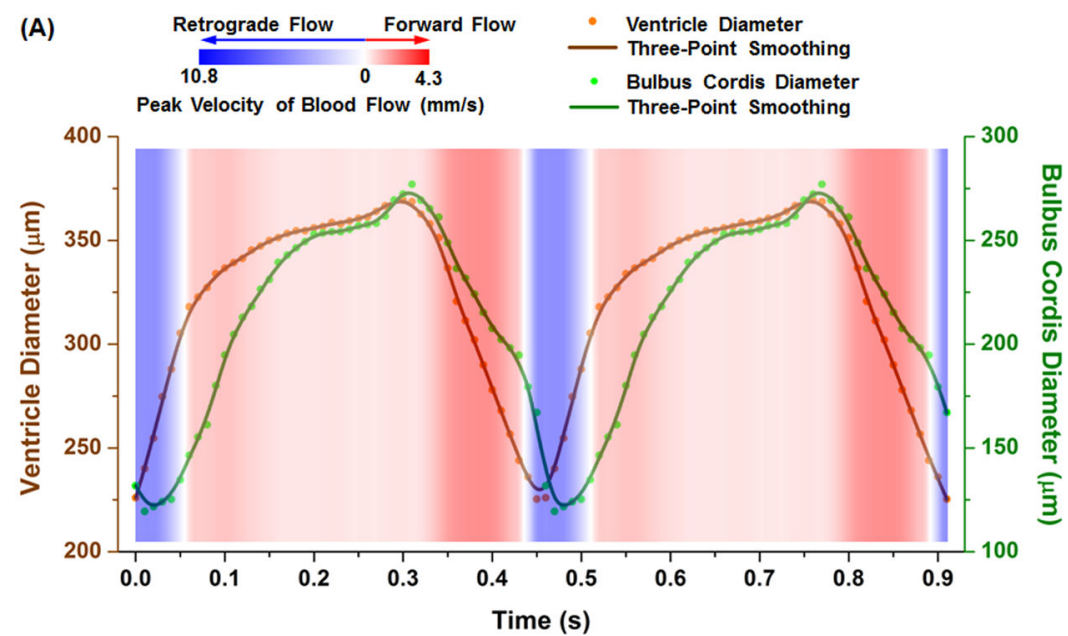

(B) $\quad \therefore$ Average of Ventricle Wall Velocity - Three-Point Smoothing Zero Line

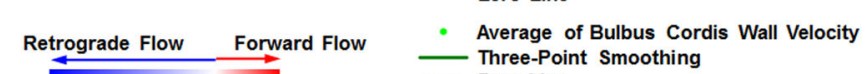
$\stackrel{\text { Three-Point Smoothing }}{\longrightarrow}$ Zero Line

Peak Velocity of Blood Flow ( $\mathrm{mm} / \mathrm{s}$ ) Wall Velocity: Positive - Relaxation

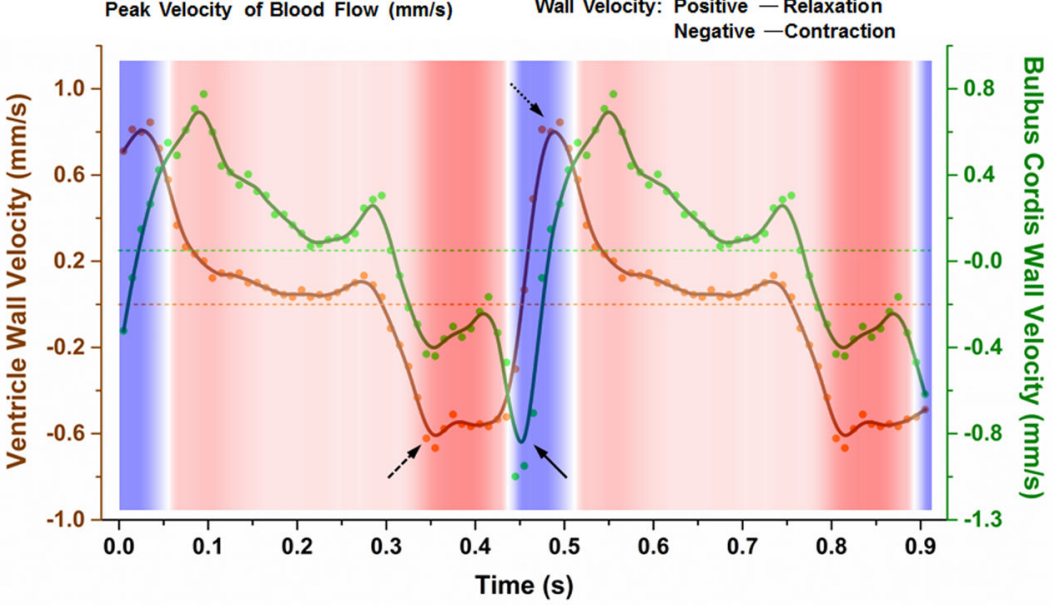

Figure 5.

Analysis of bulboventricular hemodynamics in relation to the heart wall dynamics in the central parts of the ventricle and bulbus cordis in an E9.0 mouse embryo. (A) Diameters and (B) average heart wall velocities of the ventricle and the bulbus cordis are plotted with blood flow dynamics in the bulboventricular region, showing the capability of performing combined high-resolution time-resolved cardiodynamic and hemodynamic analysis with this imaging method. The second heartbeat cycle is duplication of the first one for better visualization. 


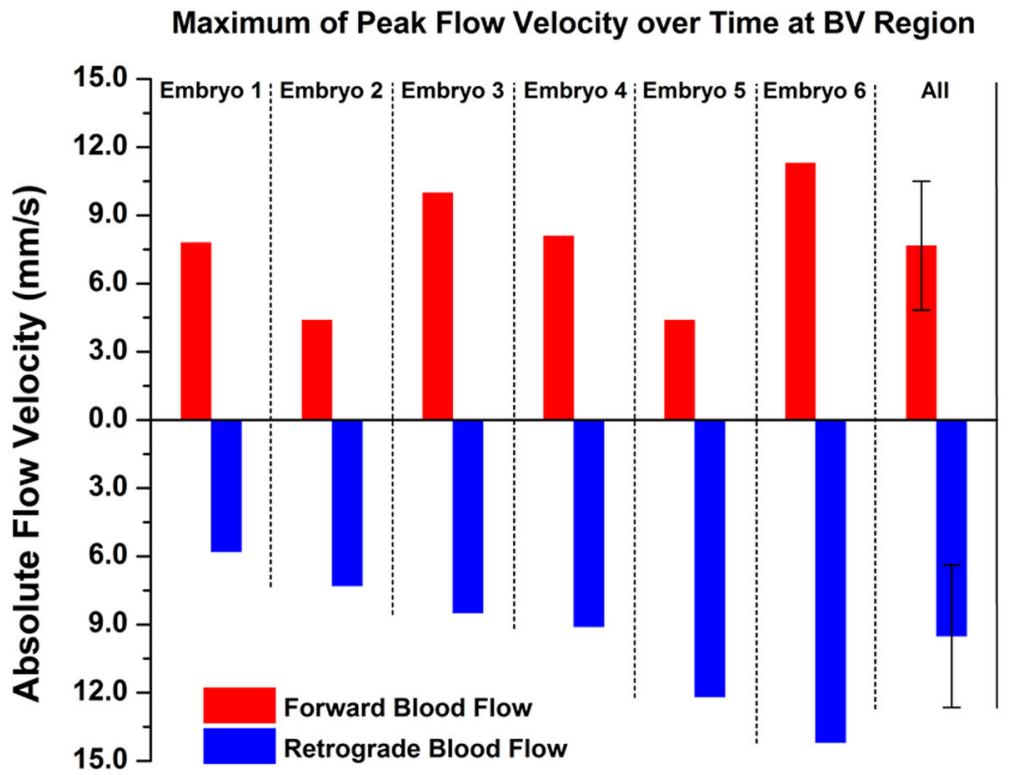

Figure 6.

Maximal peak flow velocity over one heartbeat cycle in the bulboventricular region from six E9.0 mouse embryos. 